Original Article

\title{
Long-term Survivals, Toxicities and the Role of Chemotherapy in Early-Stage Nasopharyngeal Carcinoma Patients Treated with Intensity-Modulated Radia- tion Therapy: A Retrospective Study with 15-Year Follow-up
}

\author{
Lin Wang ${ }^{1}$, Jingjing Miao ${ }^{1}$, Huageng Huang ${ }^{1}$, Boyu Chen $^{1}$, Xiao Xiao ${ }^{1}$, Manyi Zhu $^{1}$, Yingshan Liang ${ }^{1}$, Weiwei Xiao ${ }^{2}$, Shaomin Huang $^{2}$, \\ Yinglin Peng ${ }^{2}$, Xiaowu Deng ${ }^{2}$, Xing Lv' ${ }^{1}$, Weixiong Xia', Yanqun Xiang ${ }^{1}$, Xiang Guo ${ }^{1}$, Fei Han², Chong Zhao \\ Departments of ${ }^{1}$ Nasopharyngeal Carcinoma and ${ }^{2}$ Radiotherapy, Sun Yat-sen University Cancer Center, State Key Laboratory of Oncology \\ in South China, Collaborative Innovation Center for Cancer Medicine, Guangdong Key Laboratory of Nasopharyngeal Carcinoma Diagnosis and \\ Therapy, Guangzhou, China
}

Purpose This study was aimed to investigate long-term survivals and toxicities of early-stage nasopharyngeal carcinoma (NPC) in endemic area, evaluating the role of chemotherapy in stage II patients.

Materials and Methods Totally 187 patients with newly diagnosed NPC and restaged American Joint Committee on Cancer/ International Union Against Cancer 8th T1-2NO-1M0 were retrospectively recruited. All received intensity-modulated radiotherapy (IMRT) \pm chemotherapy (CT) from 2001 to 2010.

Results With 15.7-year median follow-up, 10-year locoregional recurrence-free survival, distant metastasis-free survival (DMFS), disease-specific survival (DSS), and overall survival (OS) were 93.3\%, 93.5\%, 92.9\% and 88.2\%, respectively. Multivariable analyses showed cervical lymph nodes positive and pre-treatment prognostic nutritional index $\geq 52.0$ could independently predict DMFS $(p=0.036$ and $p=0.011)$, DSS $(p=0.014$ and $p=0.026)$, and OS $(p=0.002$ and $p<0.001)$; Charlson comorbidity index $<3$ points could predict DSS $(p=0.011)$; age $>45$ years $(p=0.002)$ and pre-treatment lactate dehydrogenase $\geq 240 \mathrm{U} / \mathrm{L}(\mathrm{p}<0.001)$ predicted $0 S$. No grade 4 late toxicity happened; grade 3 late toxicities included subcutaneous fibrosis (4.3\%), deafness or otitis (4.8\%), skin dystrophy (2.1\%), and xerostomia (1.1\%). No differences on survivals were shown between IMRT+CT vs. IMRT alone in stage II patients, even in T2N1M0 ( $p>0.05$ ). Unsurprising, patients in IMRT+CT had more acute gastrointestinal reaction, myelosuppression, mucositis, late ear toxicity, and cranial nerve injury (all $p<0.05$ ) than IMRT alone group.

Conclusion Superior tumor control and satisfying long-term outcomes could be achieved with IMRT in early-stage NPC with mild late toxicities. As CT would bring more toxicities, it should be carefully performed to stage II patients.

Key words Early-stage, Nasopharyngeal carcinoma, Intensity-modulated radiation therapy, Chemotherapy, Long-term outcomes, Toxicity

\section{Introduction}

Nasopharyngeal carcinoma (NPC) is a prevalent head and neck malignancy in the South-eastern part of Asia, especially in the middle and western regions of Guangdong province [1]. Given its exquisite anatomical location and high radiosensitivity, radiotherapy is the treatment backbone for NPC. Especially with the advent of intensity-modulated radiotherapy (IMRT), dose intensity could be optimized to the gross tumor and subclinical target volumes by sharp dose fall-offs, which has been translated into improved tumor control $[2,3]$. The 5-year locoregional recurrence-free survival (LRRFS) rate has improved from $70 \%$ during the era of conventional radiotherapy (RT) to more than $90 \%$ with IMRT for the earlystage NPC [4-7], but the long-term outcomes and late toxicities in early-stage NPC patients treated by IMRT are still in absence.

According to the latest National Comprehensive Cancer Network clinical practice guideline in oncology (NCCN guidelines) version 1.2020, RT alone is recommended for stage I patients, and uniform chemoradiotherapy strategy is recommended for stage II-IV patients. But there are many differences among stage II-IV NPC patients. Foremost, the range of tumor invasion is quite disparate, no bone invasion
Correspondence: Chong Zhao

Department of Nasopharyngeal Carcinoma, Sun Yat-sen University Cancer Center 651 Dongfeng East Road, Guangzhou 510060, China

Tel: 86-13902206160 E-mail: gzzhaochong@hotmail.com

Received January 21, 2021 Accepted June 4, 2021

Published Online June 7, 2021
Co-correspondence: Fei Han

Department of Radiotherapy, Sun Yat-sen University Cancer Center,

651 Dongfeng East Road, Guangzhou 510060, China

Tel: 86-13822113698 E-mail: hanfei@sysucc.org.cn 
in stage II patients indicating a relative limited tumor scope [8]. Second, only N0 and N1 could be classified into stage II, without the existence of bilateral, lower neck, or huge $(>6 \mathrm{~cm})$ lymph nodes metastases [8]. The more advanced tumor invasion the higher occurrence of treatment failures. Therefore, the treatment failures, including locoregional recurrence and distant metastasis, are less likely to occur in stage II NPC patients than that in stage III-IV patients. Considering the above-mentioned characteristics, it seems inappropriate to perform uniform therapeutic schedule to stage II and stage III-IV NPC patients. Besides, the role of chemotherapy in stage II NPC patients has always been under debate. Some of the previous studies thought the T2N1M0 or T1-2N1M0 NPC patients could obtain the benefit from chemotherapy under IMRT while the other stage II patients couldn't $[7,9,10]$. Most of these studies were retrospective and only reporting the 3- or 5-year survivals. Only one retrospective study had 93-month median follow-up time, which showed chemotherapy could improve T1-2N1M0 patients' locoregional control in nonendemic area [10]. And in the latest published multicenter phase II randomized trial for stage II NPC patients, the author recruited 84 patients and found that concurrent chemotherapy plus IMRT did not improve patient's disease control and 5-year survivals [11].

In this study, we retrospectively collected stage I-II NPC patients who received IMRT and had more than 15-year median follow-up in Sun Yat-sen University Cancer Center (SYSUCC), reporting the 10-year survivals and toxicities of these early-stage NPC patients in endemic area, and investigating the role of chemotherapy for stage II patients.

\section{Materials and Methods}

\section{Patient selection}

This study was approved by the Clinical Research Ethics

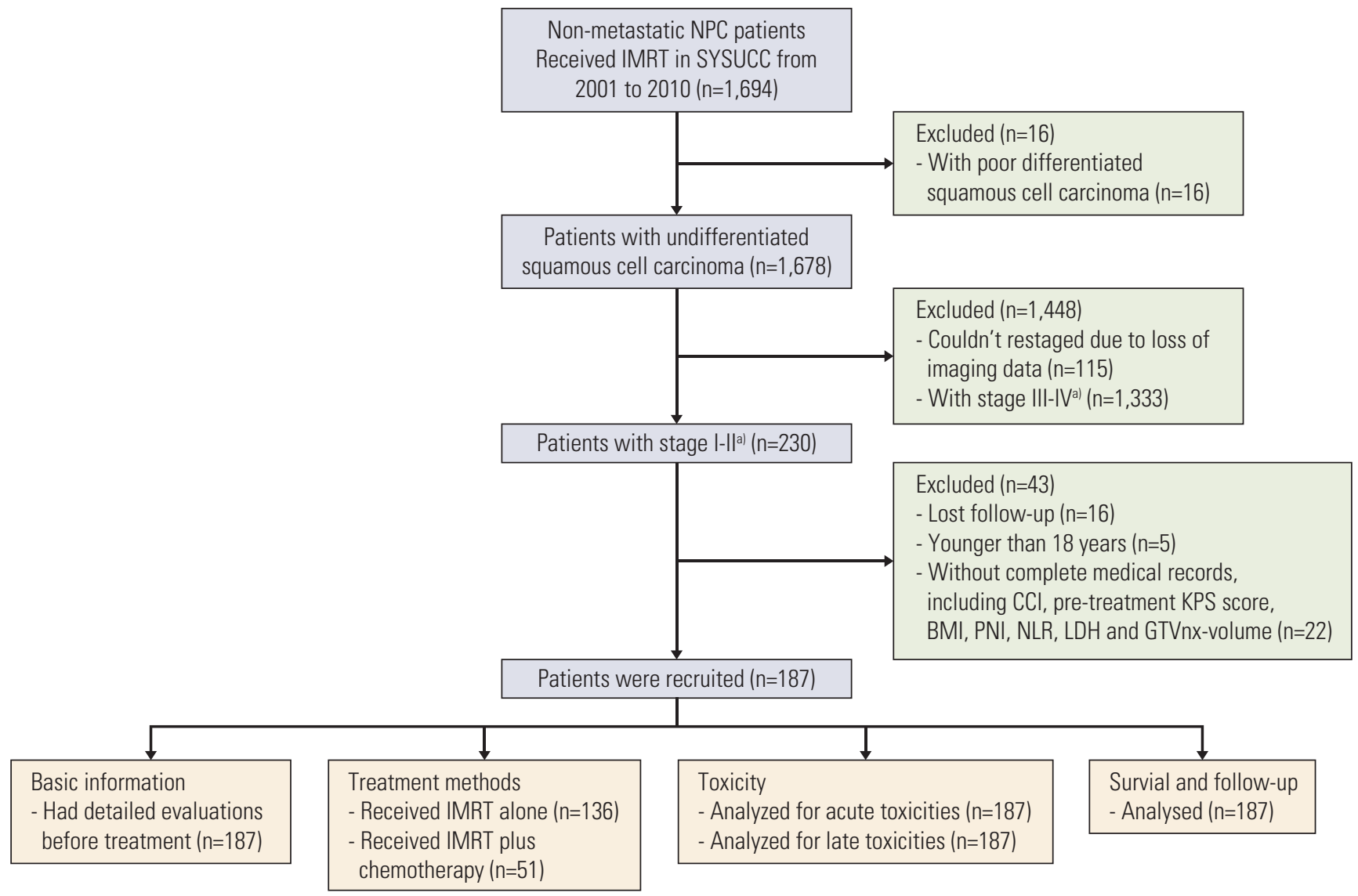

Fig. 1. Trial profile. BMI, body mass index; CCI, Charlson comorbidity index; GTVnx, gross tumor volume of nasopharynx; IMRT, intensity-modulated radiation therapy; KPS, Karnofsky performance status; LDH, lactate dehydrogenase; NLR, neutrophil to lymphocyte ratio;

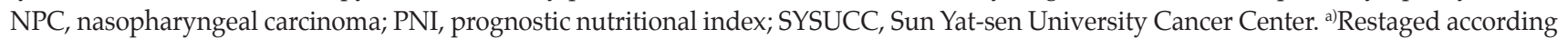
to the American Joint Committee on Cancer, 8th edition. 
Table 1. Demographic and clinical characteristics in this cohort

$\begin{array}{ll}\text { Characteristic No. }(\%) & \text { No }\end{array}$

\begin{tabular}{|c|c|}
\hline Age, median (range, yr) & $45(21-76)$ \\
\hline \multicolumn{2}{|l|}{ Sex } \\
\hline Male & $139(74.3)$ \\
\hline Female & $48(25.7)$ \\
\hline \multicolumn{2}{|l|}{ KPS score } \\
\hline 80 points & $12(6.4)$ \\
\hline 90 points & $173(92.5)$ \\
\hline 100 points & $2(1.1)$ \\
\hline \multicolumn{2}{|l|}{ WHO category } \\
\hline Keratinizing, undifferentiated & $20(10.7)$ \\
\hline Non-keratinizing, undifferentiated & $167(89.3)$ \\
\hline \multicolumn{2}{|l|}{ T category } \\
\hline $\mathrm{T} 1$ & $71(38.0)$ \\
\hline $\mathrm{T} 2$ & $116(62.0)$ \\
\hline \multicolumn{2}{|l|}{ N category ${ }^{a)}$} \\
\hline N0 & $64(34.2)$ \\
\hline N1 & $123(65.8)$ \\
\hline \multicolumn{2}{|l|}{ TNM stage $^{\mathrm{a})}$} \\
\hline I & $41(21.9)$ \\
\hline II & $146(78.1)$ \\
\hline \multicolumn{2}{|l|}{ GTVnx-volume $\left(\mathrm{cm}^{3}\right)$} \\
\hline$\leq 12.0$ & $92(49.2)$ \\
\hline$>12.0$ & $95(50.8)$ \\
\hline \multicolumn{2}{|l|}{ CLNs positive } \\
\hline No & $69(36.9)$ \\
\hline Yes & $118(63.1)$ \\
\hline \multicolumn{2}{|l|}{ RLNs positive } \\
\hline No & $102(54.5)$ \\
\hline Yes & $85(45.5)$ \\
\hline \multicolumn{2}{|l|}{ Treatment methods } \\
\hline $\mathrm{IMRT}+\mathrm{CT}$ & $51(27.3)$ \\
\hline IMRT alone & $136(72.7)$ \\
\hline \multicolumn{2}{|l|}{ CCI (points) } \\
\hline$\leq 3$ & $121(64.7)$ \\
\hline$>3$ & $66(35.3)$ \\
\hline \multicolumn{2}{|l|}{ Pre-treatment BMI $\left(\mathrm{kg} / \mathrm{m}^{2}\right)$} \\
\hline$<18.5$ & $12(6.4)$ \\
\hline$\geq 18.5$ & $175(93.6)$ \\
\hline \multicolumn{2}{|l|}{ Pre-treatment PNI } \\
\hline$<52.0$ & $38(20.3)$ \\
\hline$\geq 52.0$ & $149(79.7)$ \\
\hline \multicolumn{2}{|l|}{ Pre-treatment NLR } \\
\hline$<1.8$ & $68(36.4)$ \\
\hline$\geq 1.8$ & $119(63.6)$ \\
\hline
\end{tabular}

(Continued)

Review Committee of SYSUCC and performed in accordance with the Declaration of Helsinki and Good Clinical Practice Guidelines. Totally, 187 stage I-II NPC patients who under-
Table 1. Continued

$\begin{array}{lc}\text { Characteristic } & \text { No. }(\%) \\ \text { Pre-treatment LDH (U/L) } & \\ <240 & 177(94.7) \\ \geq 240 & 10(5.3)\end{array}$

BMI, body mass index; CCI, Charlson comorbidity index; CLNs, cervical lymph nodes; CT, chemotherapy; GTVnx, gross tumor volume of nasopharynx; IMRT, intensity-modulated radiation therapy; KPS, Karnofsky performance status; LDH, lactate dehydrogenase; NLR, neutrophil to lymphocyte ratio; PNI, prognostic nutritional index; RLNs, retropharyngeal lymph nodes; WHO, World Health Organization. a) According to the American Joint Committee on Cancer, 8th edition.

went IMRT at SYSUCC were retrospectively recruited from February 2001 to September 2010 (Fig. 1). All patients had histologically confirmed World Health Organization (WHO) type II-III NPC and complete medical records. Patients who were $<18$ years, receiving conventional RT, previous or coexisting cancers other than NPC were excluded. All patients had detailed evaluations before treatment, including physical examination, Karnofsky performance status evaluation, hematologic and biochemical analyses, chest X-ray, abdominal ultrasound, contrast-enhanced computed tomography and/or magnetic resonance imaging. And all patients were restaged according to the American Joint Committee on Cancer/International Union Against Cancer (AJCC/UICC) stage classification system, 8th edition [8].

\section{Clinical data collection}

Demographic and clinical characteristics, including age, sex, Charlson comorbidity index (CCI), WHO histological type, TNM stage, tumor volume of nasopharynx, cervical lymph nodes (CLNs) and retropharyngeal lymph nodes metastasis, pre-treatment body mass index, pre-treatment prognostic nutritional index (PNI), pre-treatment neutrophil to lymphocyte ratio, pre-treatment lactate dehydrogenase (LDH), treatment methods, acute and late toxicities, outcomes and causes of death, were reviewed from the medical records. The CCI contained both advancing ages and 19 medical conditions with a weighted score based on the relative mortality risk [12], which has proven to be a reliable and valid index of survival in a large sample of patients in various oncological settings [12-14]. And PNI, which is calculated based on the serum albumin concentration and total lymphocyte count in the peripheral blood, is known to be an indicator of both the nutritional and immune status of cancer patients [15]. In recent years, many reports have shown that the PNI could be used as a prognostic marker in patients with various malignancies [15-19]. The acute and late toxici- 
A

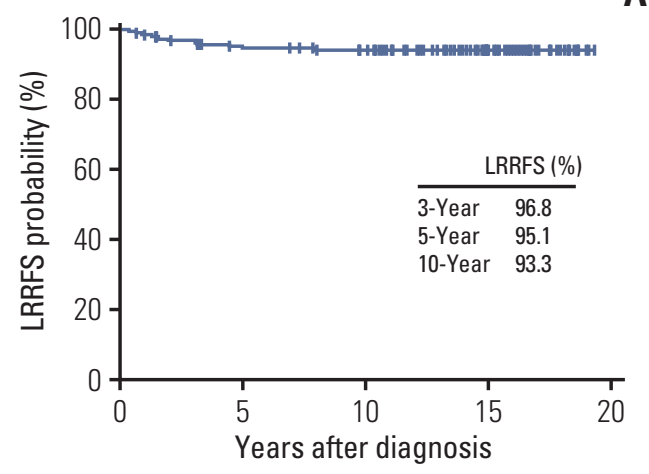

No. at risk $187 \quad 166 \quad 157 \quad 95 \quad 0$

C

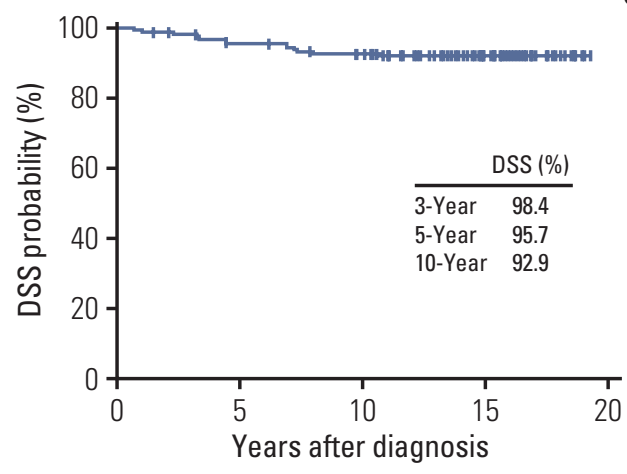

No. at risk $187 \quad 174 \quad 164 \quad 98 \quad 0$

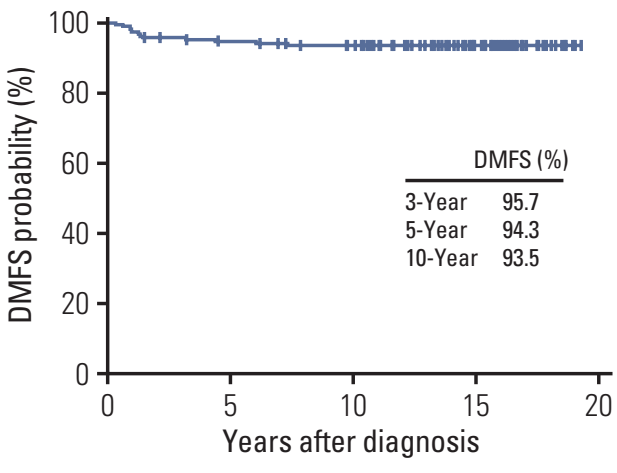

$\begin{array}{llllll}\text { No. at risk } & 187 & 172 & 162 & 97 & 0\end{array}$
B

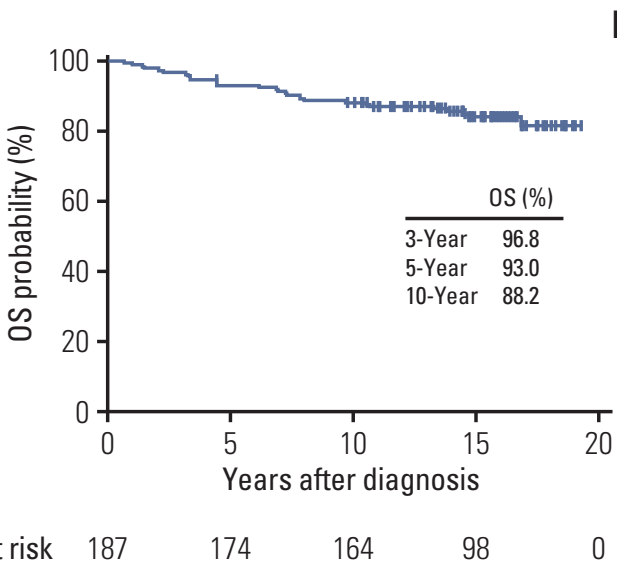

Fig. 2. Survival curves of the whole cohort: locoregional recurrence-free survival (LRRFS) (A), distant metastasis-free survival (DMFS) (B), disease-specific survival (DSS) (C), and overall survival (OS) (D).

ties were graded according to the Radiation Therapy Oncology Group radiation morbidity scoring criteria [20].

\section{Treatments}

All patients received IMRT, which was delivered with a linear accelerator (Varian, Palo Alto, CA) using $6 \mathrm{MV}$ photons. The prescribed doses were 68-70 Gy/30 fractions (fr) to the gross tumor volume of nasopharynx (GTVnx), 60-66 $\mathrm{Gy} / 30 \mathrm{fr}$ to the gross tumor volume of metastatic lymph nodes (GTVnd), $60 \mathrm{~Gy} / 30 \mathrm{fr}$ to the high-risk clinical target volume (CTV1), and $54 \mathrm{~Gy} / 30$ fr to the low-risk clinical target volume (CTV2), respectively. All of the following information has been detailed described in our previous published study: the IMRT technique, delineation method of the target volumes, dose limitation to the target volumes and organs at risk [21].

All stage I patients received IMRT alone, except one receiving concurrent chemotherapy for relatively large tumor volume $\left(G T V n x-\right.$ volume $\left.=30.4 \mathrm{~cm}^{3}\right)$. Fifty patients with stage
II disease received chemotherapy, 36 of them undertook concurrent chemotherapy (cisplatin, $80-100 \mathrm{mg} / \mathrm{m}^{2} /$ day, intravenous infusion over 2 hours on days 1 and 22), three received cisplatin-based induction chemotherapy (IC), and 11 patients received cisplatin-based IC plus concurrent cisplatin chemotherapy.

\section{Follow-up}

All patients were followed up every 3 months during the first 3 years, every 6 months during the 4 th and 5 th year then annually thereafter, with routine physical examination and nasopharyngoscopy. The hematologic and biochemical analyses, chest X-ray, abdominal ultrasound, and contrastenhanced computed tomography and/or magnetic resonance imaging were performed 3 months, 6 months and yearly after IMRT. Further investigations would be arranged when clinically indicated. All patients were followed up until May 31, 2020, or death from any cause. 


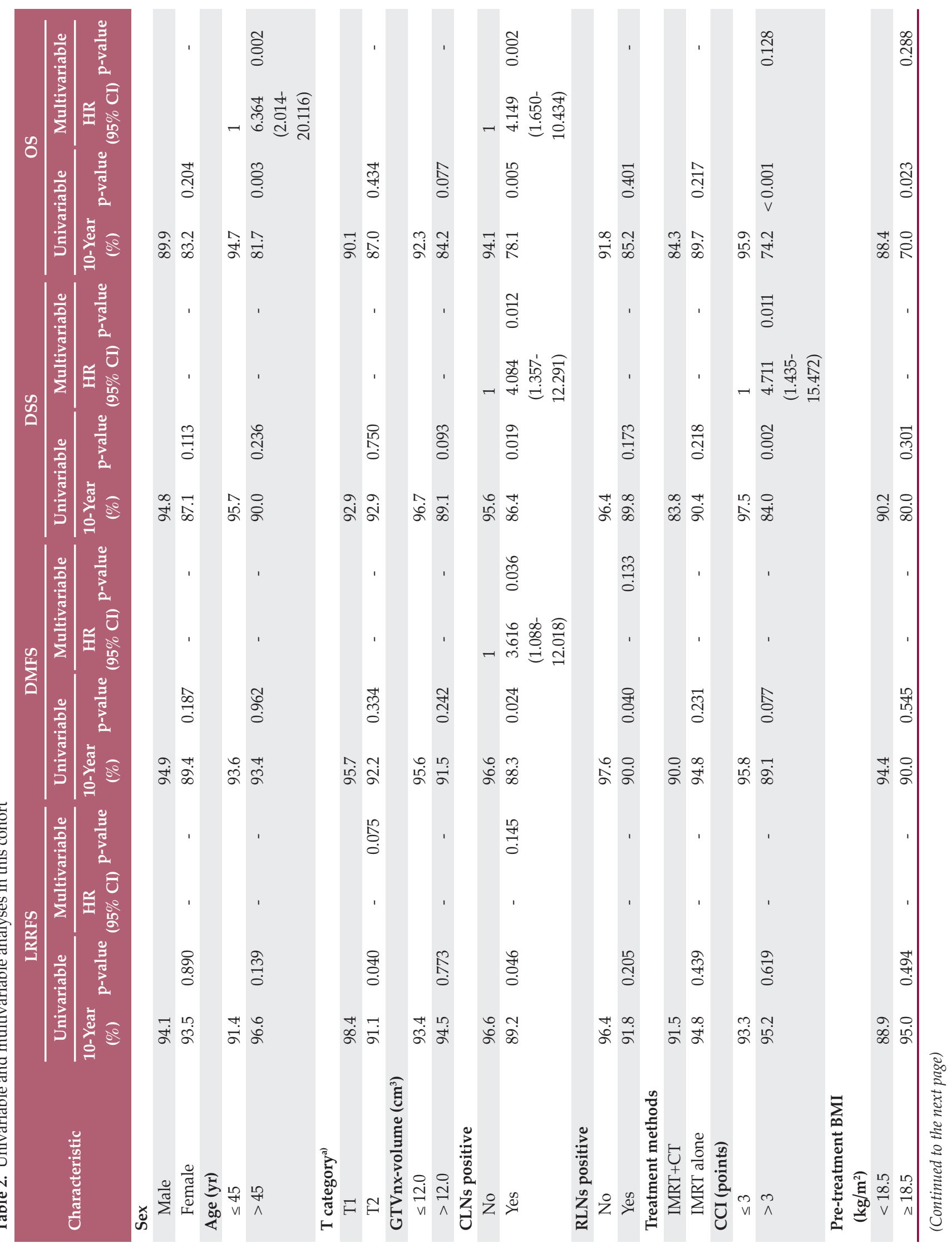




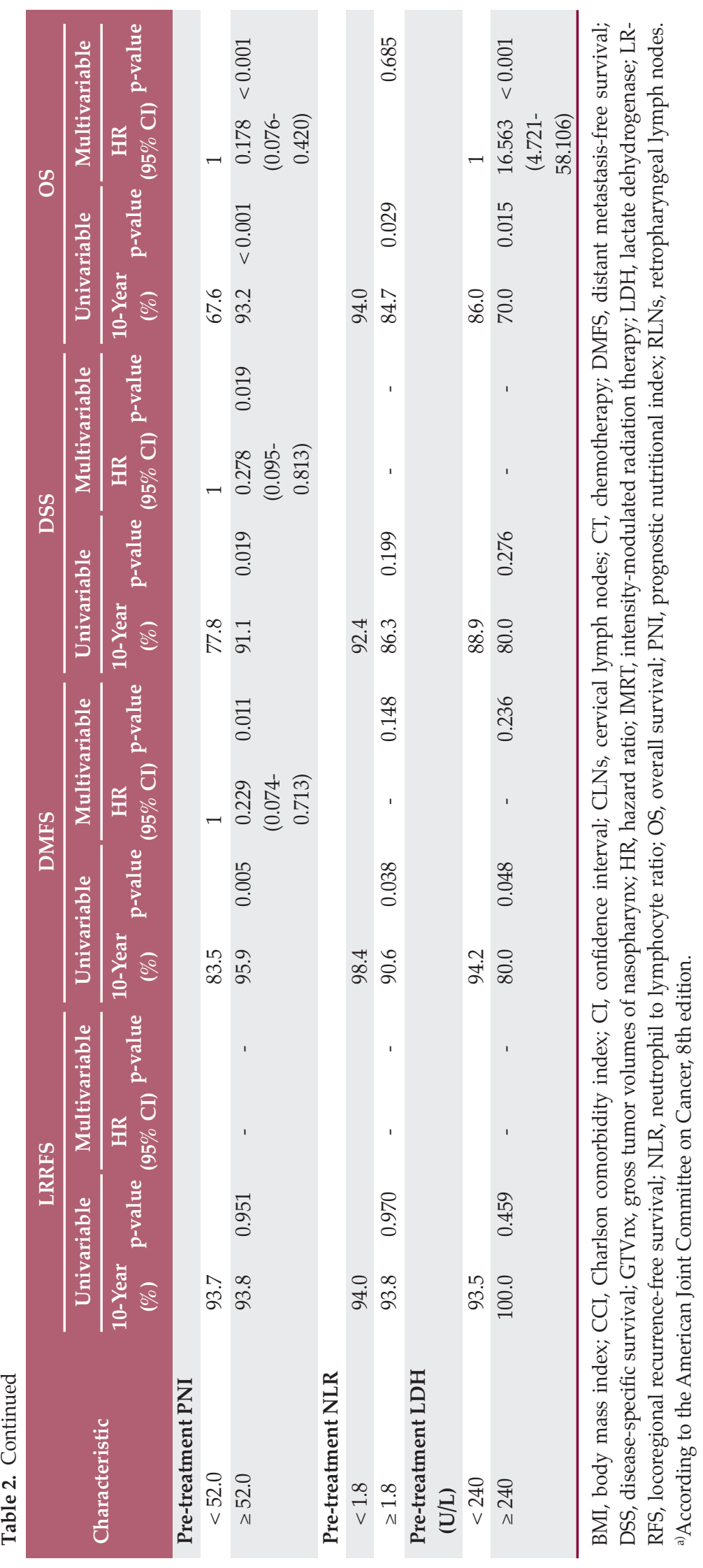


Table 3. Incidence of acute and late toxicities

\begin{tabular}{|c|c|c|c|c|c|}
\hline Any event & All grades & Grade 1 & Grade 2 & Grade 3 & Grade 4 \\
\hline \multicolumn{6}{|l|}{ Acute toxicities } \\
\hline Mucositis & $187(100)$ & $39(20.9)$ & $104(55.6)$ & $44(23.5)$ & 0 \\
\hline Radiodermatitis & $186(99.5)$ & $150(80.2)$ & $36(19.3)$ & 0 & 0 \\
\hline Xerostomia & $180(96.3)$ & $75(40.1)$ & $102(54.5)$ & $3(1.6)$ & 0 \\
\hline Gastrointestinal reaction & $103(55.1)$ & $52(27.8)$ & $31(16.6)$ & $20(10.7)$ & 0 \\
\hline Ear & $79(42.2)$ & $77(41.2)$ & $2(1.1)$ & 0 & 0 \\
\hline Leukopenia & $64(34.2)$ & $33(17.6)$ & $25(13.4)$ & $5(2.7)$ & $1(0.5)$ \\
\hline Neutropenia & $33(17.6)$ & $16(8.6)$ & $12(6.4)$ & $4(2.1)$ & $1(0.5)$ \\
\hline Anemia & $18(9.6)$ & $13(7.0)$ & $4(2.1)$ & $1(0.5)$ & 0 \\
\hline Thrombocytopenia & $14(7.5)$ & $7(3.7)$ & $4(2.1)$ & $2(1.1)$ & $1(0.5)$ \\
\hline Hepatotoxicity & $4(2.1)$ & $4(2.1)$ & 0 & 0 & 0 \\
\hline Nephrotoxicity & 0 & 0 & 0 & 0 & 0 \\
\hline \multicolumn{6}{|l|}{ Late toxicities } \\
\hline Subcutaneous fibrosis & $168(89.8)$ & $102(54.5)$ & $58(31.0)$ & $8(4.3)$ & 0 \\
\hline Deafness or otitis & $135(72.2)$ & $86(46.0)$ & $40(21.4)$ & $9(4.8)$ & 0 \\
\hline Xerostomia & $91(48.7)$ & $76(40.6)$ & $13(7.0)$ & $2(1.1)$ & 0 \\
\hline Skin dystrophy & $88(47.1)$ & $66(35.3)$ & $18(9.6)$ & $4(2.1)$ & 0 \\
\hline Trismus & $9(4.8)$ & $6(3.2)$ & $3(1.6)$ & 0 & 0 \\
\hline Cranial nerve injury & $6(3.2)$ & $6(3.2)$ & 0 & 0 & 0 \\
\hline Temporal lobe necrosis & $3(1.6)$ & $3(1.6)$ & 0 & 0 & 0 \\
\hline Eye & 0 & 0 & 0 & 0 & 0 \\
\hline Larynx & 0 & 0 & 0 & 0 & 0 \\
\hline Mandible & 0 & 0 & 0 & 0 & 0 \\
\hline
\end{tabular}

Values are presented as number $(\%)$.

\section{Statistical analysis}

Patients' demographics and clinical characteristics were summarized as frequency and percentage for categorical variables, and median with interquartile range for continuous variables. Patients' long-term outcomes, including LRRFS (freedom from documented locoregional recurrence or death from any cause), distant metastasis-free survival (DMFS; freedom from documented distant metastasis or death from any cause), disease-specific survival (DSS; freedom from documented death from NPC-related cause or last followup) and overall survival (OS; freedom from documented death from any cause or last follow-up), were estimated using the Kaplan-Meier method and compared with the logrank test. A two-sided p-value of $<0.05$ and a $95 \%$ confidence interval that did not include one was considered significant. The Cox proportional hazard model using forward stepwise elimination procedure to remove variables with a $p$-value of $\geq 0.05$ was used in multivariate analysis to determine the prognostic significance of variables. Statistical analyses were performed using the SPSS software program ver. 22.0 (IBM Corp., Armonk, NY) and the figures were drawn by GraphPad Prism 8 (GraphPad Software Inc., San Diego, CA).

\section{Results}

\section{Patient characteristics and radiotherapy dosimetry}

From February 2001 to September 2010, 187 patients were enrolled, with the detailed demographic and clinical characteristics shown in Table 1 . After being restaged using the AJCC/UICC 8th edition staging system, 41 patients were stage I and 146 patients were stage II. Among them, $51(27.3 \%)$ patients received IMRT plus chemotherapy (IMRT+CT), the other received IMRT alone. Besides, 107 patients (57.2\%) had comorbidity except NPC. The median volumes of GTVnx, GTVnd (left), GTVnd (right), CTV1, and CTV2 were $12.2 \mathrm{~cm}^{3}$, $2.8 \mathrm{~cm}^{3}, 3.9 \mathrm{~cm}^{3}, 36.5 \mathrm{~cm}^{3}$, and $198.7 \mathrm{~cm}^{3}$, respectively. The median D95 of GTVnx, GTVnd (left), GTVnd (right), CTV1, and CTV2 were 68.8 Gy, 63.2 Gy, 63.8 Gy, 64.1 Gy, and 56.8 Gy, as shown in $\mathrm{S} 1$ Table.

\section{Long-term clinical outcomes}

With a median follow-up time of 15.7 years (range, 0.7 to 19.3 years), four patients had local recurrences (all within 5 years after IMRT), and seven suffered regional recurrences (6 within 5 years after IMRT and one on 7.7 years after IMRT). Twelve patients experienced distant metastases (10 
A

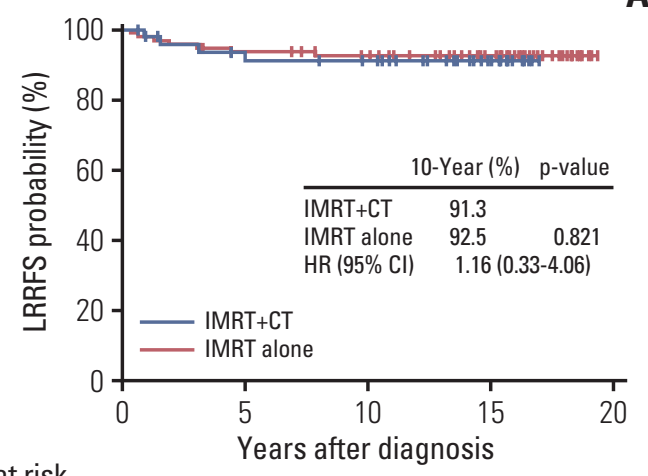

$\begin{array}{rrrrrr}\text { No. at risk } & & & & & \\ \text { IMRT+CT } & 50 & 41 & 39 & 18 & 0 \\ \text { IMRT alone } & 96 & 86 & 80 & 61 & 0\end{array}$

C

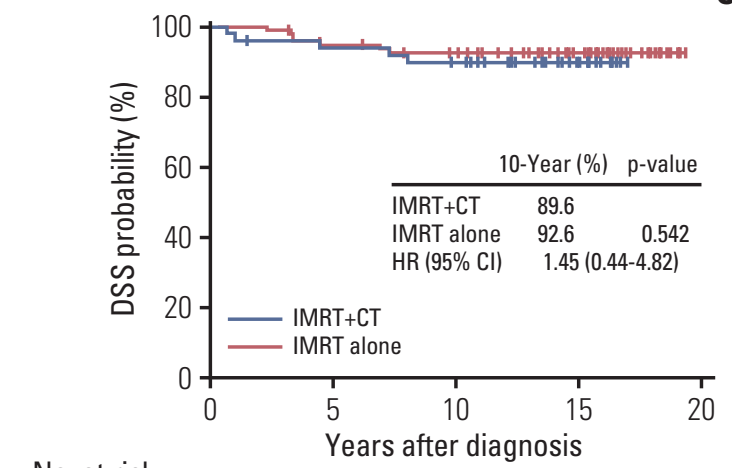

No. at risk

$\begin{array}{llllll}\text { IMRT+CT } & 50 & 45 & 42 & 18 & 0\end{array}$

IMRT alone $\quad 96 \quad 90 \quad 84 \quad 64 \quad 0$
A

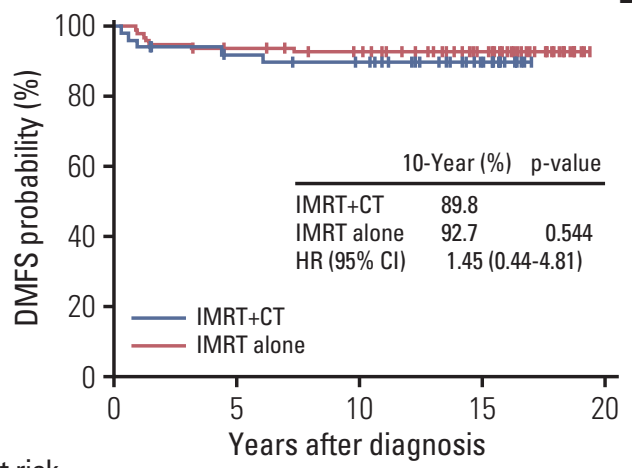

\begin{tabular}{rrrrrr} 
No. at risk & \multicolumn{5}{l}{} \\
IMRT+CT & 50 & 41 & 41 & 18 & 0 \\
IMRT alone & 96 & 89 & 83 & 63 & 0
\end{tabular}

Fig. 3. Survival curves of IMRT $+\mathrm{CT}$ vs. IMRT alone in the stage II nasopharyngeal carcinoma patients: locoregional recurrence-free survival (LRRFS) (A), distant metastasis-free survival (DMFS) (B), disease-specific survival (DSS) (C), and overall survival (OS) (D). CI, confidence interval; $\mathrm{CT}$, chemotherapy; $\mathrm{HR}$, hazard ratio; IMRT, intensity-modulated radiation therapy.

within 5 years after IMRT, one on 5.8 , and one on 7.1 years after IMRT), including three lung metastases, three bone metastases, two liver metastases, two mediastinal lymph node metastasis, one lung and mediastinal lymph node metastases, and one with liver and bone metastases. We recorded 29 death events: 14 were NPC-related, and 15 were non-NPC-related (three secondary malignancy-related, seven non-cancer-related, and five unknown). The 10-year LRRFS, DMFS, DSS, and OS in whole cohort were $93.3 \%, 93.5 \%$, $92.9 \%$, and $88.2 \%$, respectively (Fig. 2). And the long-term outcomes of patients with different TNM stage were shown in S2 Fig.

In the multivariable analyses (Table 2), CLNs and pretreatment PNI were statistically significant in predicting DMFS (CLNs, $\mathrm{p}=0.036$; pre-treatment PNI, $\mathrm{p}=0.011$ ), DSS (CLNs, $\mathrm{p}=0.014$; pre-treatment PNI, $\mathrm{p}=0.026$ ), and OS (CLNs, $\mathrm{p}=0.002$; pre-treatment PNI, $\mathrm{p}<0.001)$. Besides, CCI could statistically predicted DSS $(\mathrm{p}=0.011)$, age and pre-treatment LDH could also predicted OS significantly ( $\mathrm{p}=0.002$ and $\mathrm{p}<$ 0.001 ). Additionally, only $T$ category was marginally significant in predicting LRRFS ( $\mathrm{p}=0.075)$.

\section{Acute and late toxicities}

The most common acute toxicities (grade 1-4) in the whole cohort included mucositis $(100.0 \%)$, radiodermatitis $(99.5 \%)$, followed by xerostomia (96.3\%). As for the grade 3-4 acute toxicity, the top three toxicities were mucositis $(23.5 \%)$, gastrointestinal reaction $(10.7 \%)$, and leukopenia $(3.2 \%)$. The grade 4 acute toxicities only included one leukopenia, one neutropenia, and one thrombocytopenia, all occurred among patients who received chemotherapy.

As for the late toxicity, the most common adverse events 


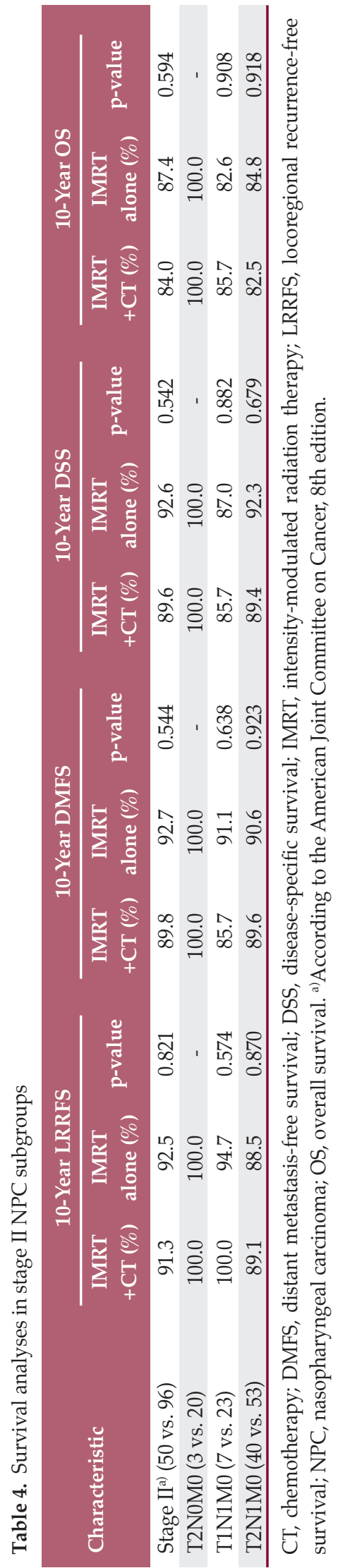

were subcutaneous fibrosis $(89.8 \%)$, deafness or otitis $(72.2 \%)$, xerostomia $(48.7 \%)$, and skin dystrophy $(47.1 \%)$. We also recorded six grade 1 cranial nerve injury and three grade 1 temporal lobe necrosis. No grade 4 late toxicities were .0 observed; and the grade 3 toxicities only included subcutaneous fibrosis $(4.3 \%)$, deafness or otitis $(4.8 \%)$, skin dystrophy $(2.1 \%)$, and xerostomia $(1.1 \%)$. The detailed acute and late toxicities were shown in Table 3.

\section{Role of chemotherapy in stage II NPC patients}

In order to verify the efficacy of chemotherapy in stage II NPC patients, we divided the stage II patients into two subgroups according to their treatment methods, with basic information shown in S3 Table. Fifty patients had chemotherapy, including 36 receiving concurrent chemoradiation therapy (CCRT), 11 receiving IC+CCRT, and 3 receiving IC+IMRT; 96 patients received IMRT alone. As shown in Fig. 3 , the 10-year LRRFS, DMFS, DSS, and OS in stage II patients treated with IMRT+CT vs. IMRT alone were $91.3 \%$ vs. $92.5 \%$ (hazard ratio [HR], 1.16; $\mathrm{p}=0.821$ ), $89.8 \%$ vs. $92.7 \%$ (HR, 1.45; $\mathrm{p}=0.544), 89.6 \%$ vs. $92.6 \%$ (HR, $1.45 ; \mathrm{p}=0.542)$, and $84.0 \%$ vs. $87.4 \%$ (HR, 1.26; $\mathrm{p}=0.594)$, respectively. In order to investigate the role of chemotherapy in T2N1M0 and T1N1M0 patients, we did subgroup analysis by dividing stage II patients with different TNM stage. And no significant differences were found between patients with IMRT+CT vs. IMRT alone in the subgroups of T2N0M0, T1N1M0, and T2N1M0 (all $\mathrm{p}>0.05$ ), as shown in Table 4.

Unsurprising, stage II patients treated with IMRT $+\mathrm{CT}$ had relatively higher incidence of gastrointestinal reaction $(\mathrm{p}=0.007)$, myelosuppression including leukopenia ( $\mathrm{p}<$ $0.001)$, neutropenia $(\mathrm{p}<0.001)$, anemia $(\mathrm{p}=0.004)$, and thrombocytopenia $(\mathrm{p}=0.005)$, and grade $3-4$ mucositis $(\mathrm{p}=0.001)$ than the IMRT alone group. As for the late toxicity, patients treated with IMRT $+\mathrm{CT}$ had significant higher incidence of deafness or otitis $(\mathrm{p}=0.002)$ and cranial nerve injury $(\mathrm{p}=0.046)$, as shown in S4 Table.

\section{Discussion}

As the solely study with over 15-year follow-up in the era of IMRT in the NPC endemic area, it showed satisfied long-term survivals for early-stage NPC with 10-year LRRFS, DMFS, and DSS over 90\% with mild late toxicity, especially for the T1-2N0M0 patients. And chemotherapy didn't improve stage II patients' tumor control and survivals in the era of IMRT, even for the T2N1M0 patients, but increased patients' acute and late toxicities.

In the era of conventional RT, the 5-year LRRFS and OS were only $73 \%-84 \%$ and $67 \%-85 \%$ among early-stage NPC 
patients [4-6]. With the advent of IMRT, superior radiation dosimetry could be delivered, which not only reduce the dose to surrounding normal tissues but also keep high radical irradiation to tumors, the tumor control has greatly improved with 5-year LRRFS over 90\% for early-stage NPC [7]. Compared with primary tumor in nasopharynx, metastatic CLNs have a higher radio-sensitivity, so lower dose was given to GTVnd. In our practical application, 60-62 Gy / 30 fr was performed to the equivocal lymph nodes (LNs), 62-64 Gy/30 fr to involved LNs with maximal axial diameter $<3 \mathrm{~cm}$, and 64-66 Gy / 30 fr to involved LNs, with maximal axial diameter $\geq 3 \mathrm{~cm}$ or central necrosis or extracapsular extension $[14,16,21]$. In this study, the 5-year LRRFS of whole cohort was $95.1 \%$ and 10-year LRRFS still reaching 93.3\%, which indicating an excellent long-term tumor control with the use of IMRT. Additionally, the 10-year DMFS and DSS were also over $90 \%$, and the 10 -year OS reached $88.2 \%$ which was far superior than that in the era of conventional RT with a 10 -year OS rate $68 \%-77 \%$ [6]. It is worth noting that some patients could suffer tumor relapse even 5 years after IMRT, one had regional recurrence and two had distant metastases beyond 5 years after IMRT in this cohort, which remind us that continuous follow-up is essential. And for these earlystage NPC patients, the CLNs positive and pre-treatment PNI could independently predict the DMFS and DSS; and CCI could predict patients' DSS. In our previous study, PNI, which represents patient's nutritional and immune status, has been proven to be an independent predictor for all stage NPC patients and could guide the treatment strategy for stage II-IV NPC patients [16]. Besides, we also found that $\mathrm{CCI}<5$ points could significantly predict the LRRFS in elderly NPC patients [14], while in this study, $\mathrm{CCI}<3$ points could independently predict NPC patients' DSS.

There were rare studies concerning the late toxicities for early-stage NPC no matter in the era of conventional RT or IMRT. In this study, we summarized patients' acute and late toxicities in detail, especially the latter. Most of patients' late toxicities were in grade $1-2$, and only $4.8 \%$ patients had grade 3 deafness or otitis, $4.3 \%$ patients had grade 3 subcutaneous fibrosis, $2.1 \%$ had grade 3 skin dystrophy and $1.1 \%$ had grade 3 xerostomia. As for the radiation-related nerve tissue damage, there were still few patients experienced grade 1 posterior cranial nerve injury with IMRT, which may be related with the severe subcutaneous fibrosis. Besides, three patients had grade 1 temporal lobe necrosis in our study whose temporal lobe irradiation were under corresponding tolerance dose, whether it is due to individual differences on temporal lobe radio-sensitivity still need further investigate.

For stage II NPC patients, whether adding chemotherapy has always been under debate from the era of conventional RT to IMRT. With the publications of the phase III randomized trial comparing CCRT vs. RT alone in stage II NPC and other retrospective studies [4-6,22], chemoradiotherapy has become the standard treatment strategy for stage II NPC in the era of conventional RT. With the widespread application of IMRT, there were mainly two opinions concerning the use of chemotherapy, some thought all of the stage II NPC didn't need chemotherapy and it couldn't improve patient's survivals but increased toxicities [23-25]; while others thought patients with T1-2N1M0 [10] or at least T2N1M0 $[7,9]$ could get benefit from chemotherapy and other subgroups needn't. In the latest published phase II trial recruited 84 stage II NPC patients, the 5-year OS, local failure-free survival, regional failure-free survival, and DMFS of the IMRT alone vs. CCRT groups were $100 \%$ vs. $94.0 \%, 93.0 \%$ vs. $89.3 \%, 97.7 \%$ vs. $95.1 \%$, and $95.2 \%$ vs. $94.5 \%$ (all $\mathrm{p}>0.05$ ). Though $80 \%$ patients $(4 / 5)$ suffering distant metastasis with or without locoregional recurrence were in T2N1M0, the author didn't perform subgroup analysis by stratifying stage II patients as T1N1M0, T2N0M0, and T2N1M0 due to the relatively small sample size and few events of distant metastasis [11]. In the subgroup analysis of our study, no significant differences were found in 10-year LRRFS, DMFS, DSS, and OS among T2N1M0 patients, which indicated that chemotherapy may not be able to benefit survival in those patients. Due to the small sample size in each subgroups of clinical stages, and uneven tumor volume and $\mathrm{N}$ staging between IMRT alone group and IMRT+CT group in all stage II patients, welldesigned prospective clinical studies are still needed to confirm this conclusion.

Similar to previous studies, we also found that adding chemotherapy increased patients' toxicities [9-11,23-26]. Due to cisplatin and other chemotherapeutic such as fluorouracil or docetaxel, more early-stage NPC patients suffered from gastrointestinal reactions, myelosuppression, and severe mucositis and leukopenia when adding chemotherapy. Even after chemoradiotherapy, the incidence of late ear toxicity, such as the deafness or otitis, was still much higher in patients who received chemotherapy. All of these acute and late adverse events would impair early-stage NPC patient's quality of life.

Besides, there are some limitations to this study. First, as we aim to investigate the long-term outcomes of the earlystage NPC patients, only 187 stage I-II NPC patients who received treatment before 2010 were recruited. Additionally, all patients were from single institution in endemic area, which should be verified in multicenter hospitals. And more importantly, multicenter randomized clinical trials with enough samples are needed to validate our results.

In summary, we report the 10-year tumor control and survivals for early-stage NPC patients in endemic area, indicating that superior tumor control and satisfying outcomes can 
be achieved with IMRT with mild toxicities. As chemotherapy would bring more acute and late toxicities for patients, so it should be carefully performed to stage II patients considering their individual factors, even for T2N1M0 patients. Multicenter randomized clinical trials should be launched to validate our conclusions, and collectively, it will motivate an optimal treatment strategy for the early-stage NPC patients.

\section{Electronic Supplementary Material}

Supplementary materials are available at Cancer Research and Treatment website (https:// www.e-crt.org).

\section{Ethical Statement}

The study was approved by the Ethics Committee of Sun Yat-sen University Cancer Center (B2020-276) and was performed in accordance with the Declaration of Helsinki and Good Clinical Practice Guidelines. All patients had signed informed consent authorizing the use of clinical data.

\section{Author Contributions}

Conceived and designed the analysis: Han F, Zhao C.

Collected the data: Wang L, Miao J, Huang H, Chen B, Xiao X, Zhu M, Liang Y.

Contributed data or analysis tools: Wang L, Miao J, Huang H, Chen B, Xiao X, Zhu M, Liang Y.

Performed the analysis: Wang L, Miao J, Huang H, Xiao W.

Wrote the paper: Wang L, Miao J, Han F, Zhao C.

Review the paper: Xiao W, Huang S, Peng Y, Deng X, Lv X, Xia W, Xiang Y, Guo X, Han F, Zhao C.

\section{Conflicts of Interest}

Conflict of interest relevant to this article was not reported.

\section{Acknowledgments}

This study was funded by the National Natural Science Foundation of China (No. 81872469); Young Teacher Foundation of Sun Yat-sen University (No. 20ykpy172); Cancer Precision Radiotherapy Spark Program of China International Medical Foundation (2019-N-1120).

The authors thank the patients and their families for their participation in this study.

\section{References}

1. Wei KR, Zheng RS, Zhang SW, Liang ZH, Li ZM, Chen WQ. Nasopharyngeal carcinoma incidence and mortality in China, 2013. Chin J Cancer. 2017;36:90.

2. O'Sullivan B, Rumble RB, Warde P; Members of the IMRT Indications Expert Panel. Intensity-modulated radiotherapy in the treatment of head and neck cancer. Clin Oncol (R Coll Radiol). 2012;24:474-87.

3. Peng G, Wang T, Yang KY, Zhang S, Zhang T, Li Q, et al. A prospective, randomized study comparing outcomes and toxicities of intensity-modulated radiotherapy vs. conventional two-dimensional radiotherapy for the treatment of nasopharyngeal carcinoma. Radiother Oncol. 2012;104:286-93.

4. Chua DT, Ma J, Sham JS, Mai HQ, Choy DT, Hong MH, et al. Improvement of survival after addition of induction chemotherapy to radiotherapy in patients with early-stage nasopharyngeal carcinoma: Subgroup analysis of two Phase III trials. Int J Radiat Oncol Biol Phys. 2006;65:1300-6.

5. Xiao WW, Han F, Lu TX, Chen CY, Huang Y, Zhao C. Treatment outcomes after radiotherapy alone for patients with early-stage nasopharyngeal carcinoma. Int J Radiat Oncol Biol Phys. 2009;74:1070-6.

6. Song $\mathrm{CH}, \mathrm{Wu} \mathrm{HG}$, Heo DS, Kim KH, Sung MW, Park CI. Treatment outcomes for radiotherapy alone are comparable with neoadjuvant chemotherapy followed by radiotherapy in early-stage nasopharyngeal carcinoma. Laryngoscope. 2008;118:663-70.

7. Su SF, Han F, Zhao C, Chen CY, Xiao WW, Li JX, et al. Long- term outcomes of early-stage nasopharyngeal carcinoma patients treated with intensity-modulated radiotherapy alone. Int J Radiat Oncol Biol Phys. 2012;82:327-33.

8. Amin MB, Edge SB, Greene FL, Byrd DR, Brookland RK, Washington MK, et al. AJCC cancer staging manual. 8th ed. New York: Springer; 2017.

9. Luo S, Zhao L, Wang J, Xu M, Li J, Zhou B, et al. Clinical outcomes for early-stage nasopharyngeal carcinoma with predominantly WHO II histology treated by intensity-modulated radiation therapy with or without chemotherapy in nonendemic region of China. Head Neck. 2014;36:841-7.

10. Ding XC, Fan PP, Xie P, Fan BJ, Yang J, Jiang LY, et al. Ten-year outcomes of intensity-modulated radiotherapy (IMRT) combine with chemotherapy versus IMRT alone for stage II nasopharyngeal carcinoma in the real-world study (RWD). Cancer Manag Res. 2019;11:8893-903.

11. Huang X, Chen X, Zhao C, Wang J, Wang K, Wang L, et al. Adding concurrent chemotherapy to intensity-modulated radiotherapy does not improve treatment outcomes for stage II nasopharyngeal carcinoma: a phase 2 multicenter clinical trial. Front Oncol. 2020;10:1314.

12. Charlson ME, Pompei P, Ales KL, MacKenzie CR. A new method of classifying prognostic comorbidity in longitudinal studies: development and validation. J Chronic Dis. 1987;40:373-83.

13. Huang Y, Chen W, Haque W, Verma V, Xing Y, Teh BS, et al. The impact of comorbidity on overall survival in elderly naso- 
pharyngeal carcinoma patients: a National Cancer Data Base analysis. Cancer Med. 2018;7:1093-101.

14. Miao J, Wang L, Zhu M, Xiao W, Wu H, Di M, et al. Reprint of long-term survival and late toxicities of elderly nasopharyngeal carcinoma (NPC) patients treated by high-totaland fractionated-dose simultaneous modulated accelerated radiotherapy with or without chemotherapy. Oral Oncol. 2019;90:126-33.

15. Ikeya T, Shibutani M, Maeda K, Sugano K, Nagahara H, Ohtani $\mathrm{H}$, et al. Maintenance of the nutritional prognostic index predicts survival in patients with unresectable metastatic colorectal cancer. J Cancer Res Clin Oncol. 2015;141:307-13.

16. Miao J, Xiao W, Wang L, Han F, Wu H, Deng X, et al. The value of the prognostic nutritional index (PNI) in predicting outcomes and guiding the treatment strategy of nasopharyngeal carcinoma (NPC) patients receiving intensity-modulated radiotherapy (IMRT) with or without chemotherapy. J Cancer Res Clin Oncol. 2017;143:1263-73.

17. Hong S, Zhou T, Fang W, Xue C, Hu Z, Qin T, et al. The prognostic nutritional index (PNI) predicts overall survival of small-cell lung cancer patients. Tumour Biol. 2015;36:3389-97.

18. Hofbauer SL, Pantuck AJ, de Martino M, Lucca I, Haitel A, Shariat SF, et al. The preoperative prognostic nutritional index is an independent predictor of survival in patients with renal cell carcinoma. Urol Oncol. 2015;33:68.

19. Jiang N, Deng JY, Ding XW, Ke B, Liu N, Zhang RP, et al. Prognostic nutritional index predicts postoperative complications and long-term outcomes of gastric cancer. World J Gastroenterol. 2014;20:10537-44.

20. Cox JD, Stetz J, Pajak TF. Toxicity criteria of the Radiation
Therapy Oncology Group (RTOG) and the European Organization for Research and Treatment of Cancer (EORTC). Int J Radiat Oncol Biol Phys. 1995;31:1341-6.

21. Miao J, Di M, Chen B, Wang L, Cao Y, Xiao W, et al. A prospective 10-year observational study of reduction of radiation therapy clinical target volume and dose in early-stage nasopharyngeal carcinoma. Int J Radiat Oncol Biol Phys. 2020;107:672-82.

22. Chen QY, Wen YF, Guo L, Liu H, Huang PY, Mo HY, et al. Concurrent chemoradiotherapy vs radiotherapy alone in stage II nasopharyngeal carcinoma: phase III randomized trial. J Natl Cancer Inst. 2011;103:1761-70.

23. Aftab O, Liao S, Zhang R, Tang N, Luo M, Zhang B, et al. Efficacy and safety of intensity-modulated radiotherapy alone versus intensity-modulated radiotherapy plus chemotherapy for treatment of intermediate-risk nasopharyngeal carcinoma. Radiat Oncol. 2020;15:66.

24. Xu T, Shen C, Zhu G, Hu C. Omission of chemotherapy in early stage nasopharyngeal carcinoma treated with IMRT: a paired cohort study. Medicine (Baltimore). 2015;94:e1457.

25. Xu C, Sun R, Tang LL, Chen L, Li WF, Mao YP, et al. Role of sequential chemoradiotherapy in stage II and low-risk stage III-IV nasopharyngeal carcinoma in the era of intensity-modulated radiotherapy: a propensity score-matched analysis. Oral Oncol. 2018;78:37-45.

26. Xu C, Zhang LH, Chen YP, Liu X, Zhou GQ, Lin AH, et al. Chemoradiotherapy versus radiotherapy alone in stage II nasopharyngeal carcinoma: a systemic review and metaanalysis of 2138 patients. J Cancer. 2017;8:287-97. 